\section{No EXSCEUS}

Sir - Stephen O'Brien and colleagues ${ }^{1}$ have performed a valuable service in cataloguing a set of highly conserved anchor loci for use in constructing comparative gene maps that are truly comparative. However, I am less impressed at the usefulness of the concept of the smallest conserved evolutionary unit segment (SCEUS).It seems to me that breaking down conserved segments into those uniquely represented in one species is likely to produce increasingly meaningless units. As more species are added to our comparative map, yesterday's SCEUS becomes today's EXSCEUS

Rather, I think the data on shared synteny should be used to build up increasingly large ancestral segments. This is most expeditiously done with reference to divergence times between taxa being compared. Segments conserved between distantly related mammals provide a real biological basis for building ancestral gene maps. Perhaps the quantity we need is not the smallest, but the oldest conserved evolutionary unit segment (OCEUS).

The difference between these two approaches is exemplified by considering the evolution of human chromosome 21 . The genes included within this section are all syntenic in cow, but are scattered among five different mouse chromosomes ${ }^{2}$, defining five SCEUS. However, in marsupial and monotreme mammals (which diverged independently from eutherian mammals about 130 and 170 million years ago respectively), human chromosome 21 genes fall into two groups ${ }^{3}$, one of which is linked to a human chromosome 3 q marker ${ }^{4}$. In both cow and mouse, human chromosome $21 \mathrm{q}$ genes are syntenic with chromosome $3 \mathrm{q}$ genes. These comparisons define two OCEUS, which include human chromosome $3 q$ as well as 21 genes. The OCEUS (but not the SCEUS), can give an account of the evolution of human chromosome 21 , suggesting that this process involved the fusion of two ancestral autosomal regions $80-130$ million years ago, the recent fission of this region into chromosome $3 \mathrm{q}$ and 21 in the primate lineage, and multiple recent rearrangements in rodents.

Jennifer A. Marshall Graves

Department of Genetics and

Human Variation,

La Trobe University,

Bundoora, Victoria 3083

Australia

References

1. O'Brien, S.J Womack, J.E Lyons, LA. Moore, K.J. Jennkins, N.A. \& Copeland, N.G. Nature Genet. 3, 103-112 (1993).

2. Threadgill, D.S., Kraus, J.P., Krawetz, S.A. \& Womack, J.E. Proc. natn. Acad. Sci. U.S.A. 88 154-158 (1991)

3. Maccarone, P., Watson, J.M., Francis, D., Kola I. \& Graves, J.A.M. Genomics 13, 1119-1124 (1991).

4. Bennett, J.H., Hayman, D.L. \& Hope, R.M. Nature 323, 59-60 (1986).

\title{
The expanded role of NF1
}

Sir - I read with interest Bernd Seizinger's recent News and Views ${ }^{1}$ of the role in tumorigenesis of $N F 1$, which is defective in neurofibromatosis type 1 . While there is clear evidence that $N F 1$ is implicated in a wider variety of tumours than had been suspected from the analysis of NF1 families, it is important to realise that the range of tumour types seen in some familial cancer syndromes may be broader than was originally thought. Epidemiological data ${ }^{2,3}$ have demonstrated that there is, for example, an excess of lung cancer in obligate carriers of retinoblastoma gene $(R B)$ mutations, which may be reflected in the incidence of lung cancer in hereditary retinoblastoma (HRB) survivors reaching adulthood. (Long term follow up of these children is less well-documented and may not yet be prolonged enough to show a large effect, but these tumours are seen in HRB survivors). The relative risk to the parents and grandparents may be as high as 15 (ref. 3). In the seven cases where histology was available $^{3}$, six of the lung cancers were small cell type. The $R B$ product is known to be aberrant in many small cell lung cancers occurring in a nonfamilial setting ${ }^{4}$. There remains some controversy as to whether transmitting unaffected relatives have a lower risk of developing nonocular cancers than affected relatives ${ }^{2,3}$.

Isolated cases of stomach, colon and breast cancers in NF1 have been reported ${ }^{5}$, and there are case reports of neuroblastoma occurring in a clinical setting more typical of NF1 (ref.6).It is difficult to infer a common genetic origin, but it is interesting to note that in neuroblastoma type IV$S$, differentiation to lesions indistinguishable from neurofibromas can occur. These tumours can be associated with overlying cutaneous pigmentation ${ }^{7}$.
Although the phenotype of NF1 may not classically include tumours such as colon, pancreatic and breast carcinoma ${ }^{8}$, it is still possible that, in a similar fashion to $H R B$, some members of NF1 families are at increased risk of suffering the commoner adult cancers.

\section{William Foulkes}

Human Immunogenetics

Laboratory,

ICRF, PO Box 123,

Lincoln's Inn Fields,

London WC2A 3PX, UK

\footnotetext{
References

1. Seizinger, B.R. Nature Genet. 3, 97-99 (1993).

2. Strong, L.C. et al. J. Natl. Cancer Inst. 73, 303311 (1984).

3. Sanders, B.M. et al. Br. J. Cancer 60, 358-365 (1989).

Harbour, J.W. etal. Science 241, 353-357 (1988)

5. Hope, D.G. \& Mulvihill, J.J. Adv. Neurol. 29, 3356 (1981).

6. Kushner, B.H. et al. J. clin. Oncol. 3, 117-120 (1985)

7. Bolande, R.P. \& Towler, W.F. Cancer 26, 167175 (1970).

8. Riccardi, V.M. New Engl. J. Mod. 305, 16171627 (1981).
} 